\title{
c-FLIP regulates autophagy by interacting with Beclin-1 and influencing its stability
}

Luana Tomaipitinca $^{1,2}$, Simonetta Petrungaro (iD ${ }^{1}$, Pasquale D’Acunzo (iD ${ }^{3,4}$, Angelo Facchiano ${ }^{5}$, Amit Dubey ${ }^{6,7}$, Salvatore Rizza iD ${ }^{8}$ Federico Giulitti ${ }^{1}$, Eugenio Gaudio ${ }^{1}$, Antonio Filippini ${ }^{1 凶}$, Elio Ziparo ${ }^{1}$, Francesco Cecconi ${ }^{2,9,10}$ and Claudia Giampietri (iD ${ }^{1 凶}$

(c) The Author(s) 2021

c-FLIP (cellular FLICE-like inhibitory protein) protein is mostly known as an apoptosis modulator. However, increasing data underline that c-FLIP plays multiple roles in cellular homoeostasis, influencing differently the same pathways depending on its expression level and isoform predominance. Few and controversial data are available regarding c-FLIP function in autophagy. Here we show that autophagic flux is less effective in C-FLIP-/- than in WT MEFs (mouse embryonic fibroblasts). Indeed, we show that the absence of c-FLIP compromises the expression levels of pivotal factors in the generation of autophagosomes. In line with the role of C-FLIP as a scaffold protein, we found that C-FLIP interacts with Beclin-1 (BECN1: coiled-coil, moesin-like BCL2-interacting protein), which is required for autophagosome nucleation. By a combination of bioinformatics tools and biochemistry assays, we demonstrate that $\mathrm{c}-\mathrm{FLIP}$ interaction with Beclin-1 is important to prevent Beclin-1 ubiquitination and degradation through the proteasomal pathway. Taken together, our data describe a novel molecular mechanism through which $c-F_{L} P_{L}$ positively regulates autophagy, by enhancing Beclin-1 protein stability.

Cell Death and Disease (2021)12:686; https://doi.org/10.1038/s41419-021-03957-5

\section{INTRODUCTION}

FLIP (FLICE-like inhibitory protein) was described for the first time as a viral factor utilized by pathogens to escape apoptotic signalling in host cells [1]. FLIP homologues were then identified in human cells and referred to as cellular FLIP (c-FLIP) [2]. Since their characterization as anti-apoptotic factors, it became clear that c-FLIP family members exert multifunctional roles in several cellular processes, as they are involved in embryonic development, cardiac hypertrophy, skeletal muscle homoeostasis, T-cell proliferation and activation of nuclear factor-K-light-chain-enhancer of activated $B$ cells and extracellular signal-regulated kinase pathways [3-7]. Apoptosis plays a key role in liver toxic injury and its enhancement via c-FLIP removal increases liver damage in vivo [8].

Thirteen distinct splice variants originate from the human CFLAR gene, but only three of them are translated into proteins: the long isoform $C-F L I P L(55 \mathrm{kDa})$ and two short isoforms, namely $\mathrm{c}-\mathrm{FLIP} \mathrm{P}_{\mathrm{R}}$ $(25 \mathrm{kDa})$ and c-FLIP $\mathrm{S}(27 \mathrm{kDa})$ [2]. In mice, only two c-FLIP isoforms are expressed: a long and a short isoform [9]. c-FLIP structure contains two death effector domains, which mediate c-FLIP recruitment at the death-inducing signalling complexes, thus competing with procaspase 8 and inhibiting apoptosis [2]. Although the short isoforms have been confirmed to only act as negative regulators of cell death, the role of $c-F L I P_{L}$ in the balance between cell death and cell survival is more intricated and controversial. Numerous lines of evidence show that $c-F L I P_{L}$ can also support apoptosis activation [10].

Differences between c-FLIP isoforms also concern their cellular localization. Although all three variants can be found in the cytosol, C-FLIP $\mathrm{L}$ is the only one that can also localize at the endoplasmic reticulum (ER), at the mitochondria associated membranes (MAMs) and in the nucleus [11, 12].

It is emerging that c-FLIP exerts pro- or anti-apoptotic functions depending on its expression levels, and that $c-F L I P_{L}$ contribution to cell death is strictly cell type-dependent and rely on relative levels of its isoforms. Indeed, the balance between all c-FLIP isoforms controls cell fate in a dose-dependent way [13, 14].

Macroautophagy (hereafter referred to as autophagy) is a highly conserved catabolic mechanism that ensures quality control of macromolecules and whole organelles. Autophagy contributes to cellular homoeostasis by regulating the turnover of cellular components and plays a pivotal role in the regulation of body metabolism $[15,16]$. Autophagy is involved in several biological processes, such as embryonic development and cellular differentiation, but also in stress-inducing responses, which enhance autophagy activation. This mechanism is triggered by nutrients

\footnotetext{
${ }^{1}$ Department of Anatomy, Histology, Forensic Medicine and Orthopedics, Sapienza University of Rome, Rome, Italy. ${ }^{2}$ Cell Stress and Survival Unit, Danish Cancer Society Research Center, Copenhagen 2100, Denmark. ${ }^{3}$ Center for Dementia Research, Nathan S. Kline Institute for Psychiatric Research, Orangeburg, NY 10962, USA. ${ }^{4}$ Department of Psychiatry, New York University School of Medicine, New York, NY 10016, USA. Istituto di Scienze dell'Alimentazione-CNR, Avellino, Italy. ${ }^{6}$ Computational Chemistry and Drug Discovery Division, Quanta Calculus Pvt Ltd, Kushinagar 274203, India. ${ }^{7}$ Department of Pharmacology, Saveetha Dental College and Hospital, Saveetha Institute of Medical and Technical Sciences, Chennai, Tamil Nadu, India. ${ }^{8}$ Redox Signaling and Oxidative Stress Group, Danish Cancer Society Research Center, Copenhagen 2100, Denmark. ${ }^{9}$ Department of Pediatric Hemato-Oncology and Cell and Gene therapy, IRCCS Bambino Gesù Children's Hospital, Rome 00143, Italy. ${ }^{10}$ Department of Biology, University of Tor Vergata, Rome 00133, Italy. ${ }^{凶}$ email: antonio.filippini@uniroma1.it; claudia.giampietri@uniroma1.it

Edited by M. Agostini
}

Received: 22 February 2021 Revised: 14 June 2021 Accepted: 15 June 2021

Published online: 08 July 2021 
deprivation, oxidative stress and pathogen invasion, and it is impaired in several human pathologies, including neurological and metabolic disorders, infections, autoimmune diseases and tumorigenesis [17-23].

When autophagy is induced, cytoplasmic regions to be targeted for degradation are incorporated into a system of forming membranes, called phagophores or isolation membranes. The expansion of the phagophores leads to closed vesicular structures, namely autophagosomes, which are transported along the microtubules to fuse with lysosomes and form the autophagolysosomes, where hydrolytic enzymes catalyse cargo degradation [24-28].

c-FLIP isoforms were also associated with autophagy. First, it has been reported that c-FLIP can prevent autophagy-related gene 3 (Atg3) from binding microtubule-associated protein light-chain 3 (LC3). As Atg3-mediated LC3 processing and lipidation is fundamental for autophagosome formation, it was proposed that c-FLIP suppresses autophagy in basal conditions [29]. On the other hand, the antagonist of c-FLIP caspase 8 was found to play a role in autophagy repression in T cells. In detail, Fas-associated protein with death domain-caspase 8 complex can localize at the expanding autophagosome, an event that leads to caspase 8 activity induction, resulting in autophagic cell death inhibition. In the same work, the authors hypothesize that differential expression of c-FLIP isoforms can modulate the activation of caspase 8 during autophagy [30]. Remarkably, other reports show a positive correlation between the overexpression of the short c-FLIP isoform (c-FLIP S and pro-autophagic vesicle formation during anti-tumour treatments [31].

We previously demonstrated that c-FLIP absence alters ER stress-dependent apoptosis in mouse embryonic fibroblasts (MEFs), promoting cell survival [32]. Considering that autophagy is one of the most important pro-survival processes activated by cells to cope with stress conditions [33], we decided to investigate the effects of c-FLIP on autophagy activation. In the present study, we show that an interplay does exist between C-FLIP $\mathrm{L}_{\mathrm{L}}$ and Beclin-1 (BECN1: coiled-coil, moesin-like BCL2-interacting protein). Beclin-1, along with Vps34 (vacuolar protein sorting) and Vps15, constitute the phosphatidylinositol-3-kinase (PI3K-III) core complex, which mediates the formation of phosphatidylinositol-3-phosphate (Ptdlns3P), a signal molecule required for membrane specialization into phagophores [26]. Beclin-1 is essential for the association of the core complex to several transient interactors, important to define the complex localization and its biological activity [34-37]. By connecting the factors modulating the kinase activity of Vps34 to their target kinase, Beclin-1 stands at the centre of PI3K-III complexes' regulation and it is widely monitored. Beclin-1 can undergo several post-translational modifications, such as phosphorylation, ubiquitination, ISGylation and acetylation, consequently affecting the autophagic process [37]. Reduced expression of Beclin-1 was found in hepatocarcinoma, as well as in intrahepatic and extrahepatic cholangiocarcinoma, indicating Beclin-1 as a possible prognosis marker [38, 39]. We here unravel a new molecular mechanism involved in Beclin-1 protein level control, demonstrating that C-FLIP $\mathrm{L}$ prevents Beclin-1 proteasomemediated degradation by decreasing Beclin-1 ubiquitination state and acts as a positive regulator of autophagic flux.

\section{RESULTS}

\section{Autophagic flux is compromised in C-FLIP-/- cells}

We previously demonstrated that the absence of c-FLIP compromises MEFs response to ER stress-dependent cell death induced by tunicamycin. [32]. As ER stress response is strictly connected to autophagy activation, we wondered whether autophagic flux was altered in c-FLIP-/- MEFs [40]. To study c-FLIP-dependent autophagic flux, we decided to analyse two well-known autophagy markers, LC3 and p62, in wild-type (WT) MEFs compared to c-
FLIP-/- MEFs. As autophagy is a highly rapid answer to stress, and LC3 II and p62 turnover may be very fast, as already described, we used bafilomycin $A 1$, an inhibitor of autophagosomes and lysosomes fusion, to block LC3 II and p62 degradation into autophagosomes [41]. Tunicamycin induced a stronger autophagy activation in WT compared to $c$-FLIP-/ - MEFs, as detected by LC3 II and p62 accumulation in bafilomycin A1-treated cells (Supplementary Fig. S1). To specifically investigate c-FLIP involvement in autophagy, we induced the mechanism in our cells using two more stimuli, widely known to trigger this process: starvation, obtained by replacing full medium with Earle's balanced salt solution (EBBS), or torin 1 treatment, a specific mammalian target of rapamycin (mTOR) inhibitor [41]. In both starvation and torin treatment (Fig. 1A), in the presence of bafilomycin $A 1$, we observed the accumulation of LC3 II and p62 only in WT MEFs, whereas no significant markers accumulation was observed in $c$ FLIP-/- MEFs.

To further corroborate these results, autophagic flux in starvation conditions was measured by immunofluorescence, by analysing the appearance of LC3-positive puncta, in bafilomycin A1-treated cells. Coherently with western blotting outcomes, quantification of LC3 puncta shows stronger accumulation in WT cells than in C-FLIP-/- cells (Fig. 1B).

Finally, we checked Atg5 expression levels in starvation conditions at different time points. Atg5 belongs to the Atg5-Atg12-Atg16 complex, required for LC3 lipidation and maturation [42]. As shown in Supplementary Fig. S2, Atg5 protein expression increases following nutrient deprivation in WT MEFs, whereas no significant differences can be observed in c-FLIP-/- cells.

This evidence leads us to conclude that c-FLIP is required to carry on autophagic flux.

\section{c-FLIP absence does not influence autophagy induction}

Based on the previous results, to understand whether the absence of c-FLIP affected autophagy by preventing the induction step of the process, we studied mTOR and Unc-51-like autophagy activating kinase (ULK1) activation state under starvation conditions at increasing time points.

When nutrient levels available are sufficient, mTOR is activated by numerous post-translational modifications, including a positive feedback mechanism based on phosphorylation by its direct target S6 kinase 1 at Ser2448 [43]. These phosphorylation levels rapidly attenuate after nutrient deprivation, leading to mTOR deactivation and removing mTOR-mediated autophagy blockage. As evident in Fig. 2, starvation reduced mTOR Ser2448 phosphorylation in both WT and c-FLIP-/- MEFs, indicating autophagy activation in both cell types.

This result was confirmed by studying ULK1, which is a direct target of mTOR. Indeed, mTOR phosphorylates ULK1 on Ser757 to inhibit autophagy [44]. After starvation, the phosphorylation on ULK1 Ser757 decreases in both WT and c-FLIP-/- MEFs (Fig. 2), demonstrating that the absence of c-FLIP does not affect the induction phase of the process.

\section{c-FLIP absence impacts the expression levels of key proteins in autophagosomes nucleation}

As we did not find any significant differences in the induction of autophagy depending on c-FLIP absence, we focused on the nucleation step of the process, in particular on the members of the PI3K-III complex, which mediates Ptdlns3P synthesis.

We thus evaluated by western blotting the levels of Beclin-1, Vps34 and the phosphorylation status of Atg14 after starvation in a $3 \mathrm{~h}$ timeframe. Beclin-1 acts as an adaptor protein, which associates to the phosphatidylinositol-3-kinase $\mathrm{Vps} 34$ regulating its cognate partners binding. This interaction is crucial to constitute the nucleation core complex and for the kinase to generate Ptdlns3P [37]. Atg14 is another partner of the complex, ensuring its correct localization at the ER. It is phosphorylated by 
A
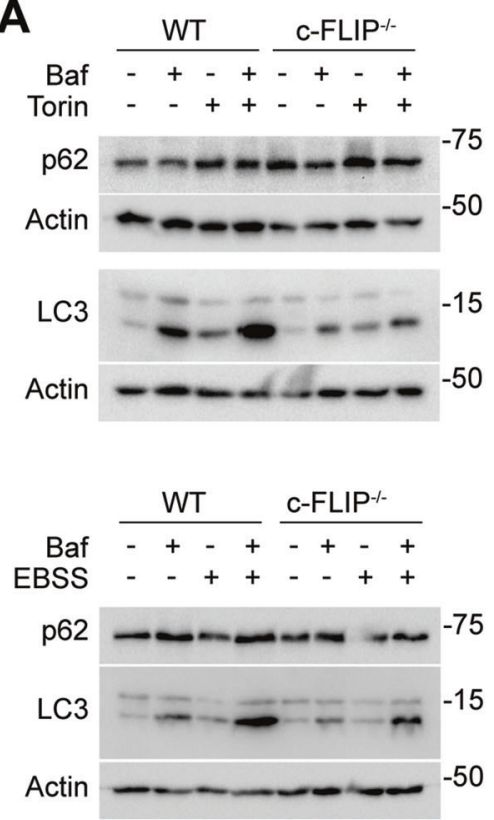
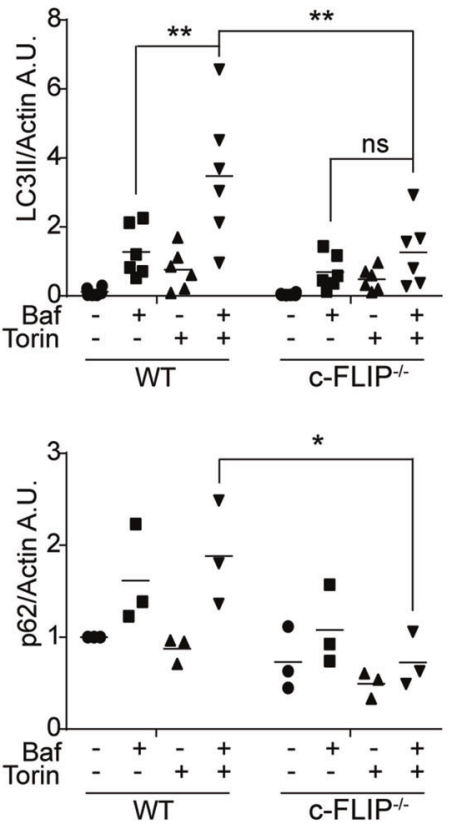
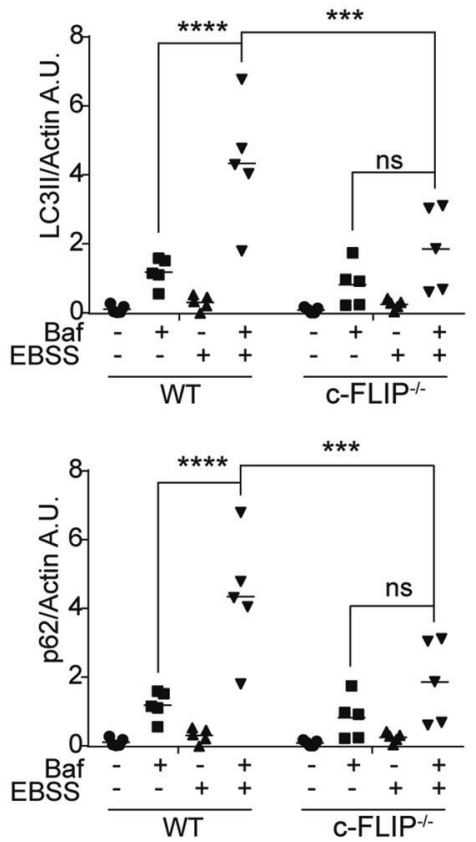

B

\begin{tabular}{c|cc}
\multicolumn{3}{c}{ WT } \\
\hline TO-PRO-3 & LC3 & $100 \mathrm{X}$ \\
\hline Ł & 4 & $\cdots$ \\
& & \\
\hline
\end{tabular}

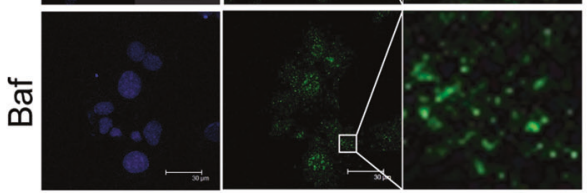

\begin{tabular}{ccc} 
C-FLIP-/- & \\
\hline TO-PRO-3 & LC3 & 100X
\end{tabular}
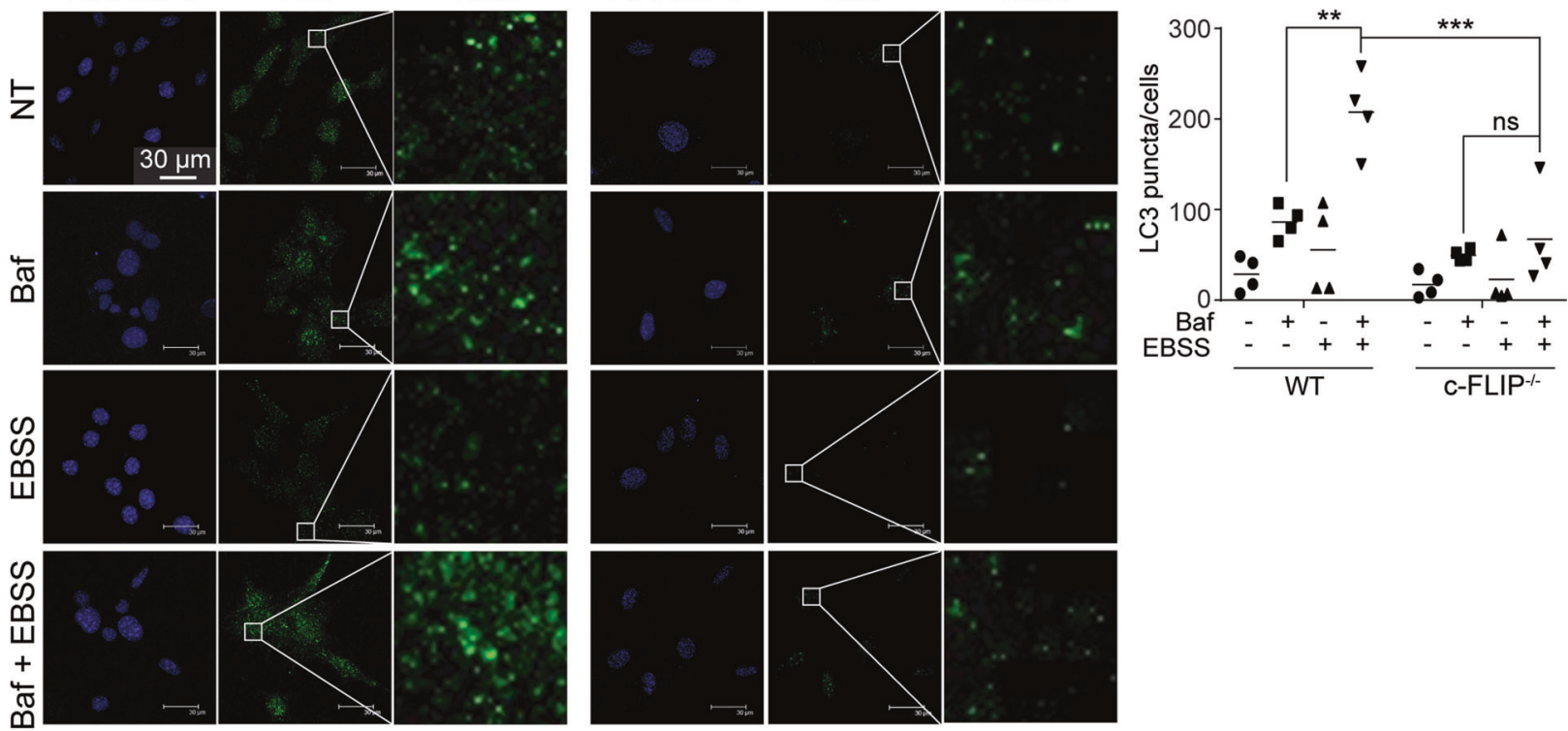

Fig. 1 Autophagic flux is compromised in c-FLIP-/- cells. A WT and c-FLIP-/- MEFs were treated with torin 1 (Tor) (250 nM) and bafilomycin A1 (Baf) $(100 \mathrm{nM})$ or cultured in a starvation medium (EBSS) and treated with bafilomycin A1 (Baf) (100 nM) for $3 \mathrm{~h}$. Autophagic flux was assessed by measuring LC3 II and p62 protein levels by western blotting. $\beta$-Actin was used as a loading control. Data shown are representative of at least three individual experiments. ${ }^{*} p<0.05,{ }^{* *} p<0.01,{ }^{* * *} p<0.001,{ }^{* * * *} p<0.0001$ determined by two-way ANOVA. B WT and $c-F L I P-/-$ MEFs were cultured in EBSS and treated with bafilomycin A1 for $3 \mathrm{~h}$. Cells were fixed and stained with anti-LC3 antibody (green) and then analysed by confocal microscopy. The images and the graph shown are representative of at least three individual experiments. ${ }^{* *} p<$ $0.01,{ }^{* *} p<0.001$ determined by two-way ANOVA. Scale bar, $30 \mu \mathrm{m}$.

ULK1 at Ser29 and this phosphorylation rate increases upon autophagy-inducing stimuli [45].

As displayed in Fig. 3A-C, nutrient deprivation results in a timedependent increase in the expression levels of these proteins in WT MEFs. Interestingly, c-FLIP $\mathrm{L}_{\mathrm{L}}$ levels are not influenced by starvation (Supplementary Fig. S3A). On the contrary, in c-FLIP-/MEFs, starvation triggers a milder effect on these factors.
Remarkably, the expression levels of Beclin-1 result to be lower in c-FLIP-/- MEFs, compared to WT cells also in untreated conditions, with a significant mean difference of $52 \%$ (Supplementary Fig. S3B).

These data indicate that c-FLIP presence is pivotal for autophagic membrane nucleation process in response to starvation. 

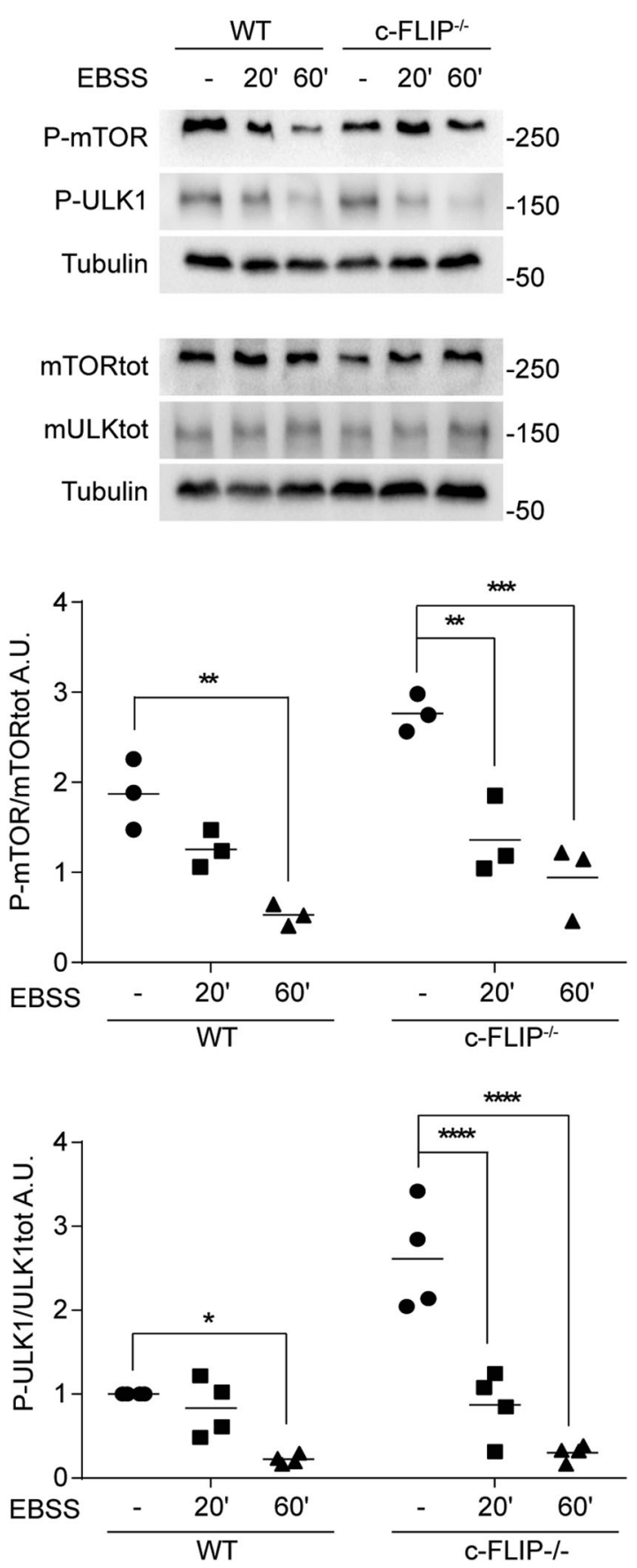

Fig. 2 WT and C-FLIP-/- MEFs were cultured in EBSS for increasing times $(20$ and $60 \mathrm{~min})$. Total $\mathrm{mTOR}$ protein and its phosphorylation levels on Ser2448, and total ULK1 and its phosphorylation levels on Ser757 were evaluated by western blotting. Graphs show the ratio of P-mTOR on mTOR total protein and of P-ULK1 on ULK1 total protein. $\alpha$-Tubulin was used as a loading control. Data shown are representative of at least three individual experiments. ${ }^{*} p<0.05,{ }^{* *} p<0.01,{ }^{* * *} p<0.001$, ${ }^{* * * *} p<$ 0.0001 determined by two-way ANOVA.

\section{c-FLIP $P_{L}$ interacts with Beclin-1 and affects Beclin-1 degradation}

As c-FLIP is a scaffold protein without known catalytic activities, it participates to cellular processes by binding other proteins and either assembling or destabilizing complexes. Therefore, we wondered whether c-FLIP could physically interact with components of the nucleation core.
It is known that Beclin-1 is a direct target of caspase 8 , and that this enzyme inactivates autophagy by cleaving Beclin-1 [46]. As caspase 8 shows high structural homology with c-FLIP and the roles of these two proteins in cellular homoeostasis are strictly connected [10], we hypothesized that Beclin-1 could be a possible interactor of c-FLIP.

To investigate this issue, HEK 293 cells were transiently transfected with two tagged constructs expressing Flag-Beclin and $\mathrm{c}-\mathrm{FLIP}_{\mathrm{L}}-\mathrm{V} 5$. We performed two co-immunoprecipitation assays on the cellular extracts and found that these two proteins interact (Fig. 4A). We also checked for an interaction between Beclin-1 and FLIPS by immunoprecipitation of c-FLIP isoforms. We detected a very low signal (if any) for Beclin-1, suggesting the absence of any biologically relevant binding between Beclin-1 and the short isoform of c-FLIP (Supplementary Fig. S4).

Interestingly, western blot analysis of HEK 293 total extracts revealed higher levels of Beclin-1 in cells in which Flag-Beclin was co-transfected with c-FLIP $\mathrm{L}_{\mathrm{L}}$ compared to cells in which Flag-Beclin was the only protein overexpressed (significant fold increase mean of 3.45) (Fig. 4A). This observation, together with data shown in Fig. 3 and Supplementary Fig. S3, where it is evident how Beclin-1 and the other nucleation proteins expression levels are lower in c-FLIP-/- compared to WT MEFs even in endogenous and basal conditions, suggested us that c-FLIP could be involved in the post-translational regulation of Beclin-1.

To verify our hypothesis, we treated both WT and C-FLIP-/MEFs with MG132, which binds the active site of the $26 \mathrm{~S}$ proteasome complex, thus blocking protein degradation [47] or bafilomycin A1 to prevent autophagosomes turnover. Indeed, Beclin-1 significantly accumulates with MG132 in c-FLIP-/- MEFs but not when using bafilomycin, whereas no differences can be observed in WT cells between the treated and the untreated samples (Fig. 4B). Consequently, we wondered if Beclin-1 ubiquitination state was influenced by $\mathrm{C}-\mathrm{FLIP}_{\mathrm{L}}$. To address this issue, we transfected HEK 293 cells with a Flag-Beclin-expressing plasmid with or without a second plasmid expressing C-FLIP $\mathrm{P}_{\mathrm{L}} \mathrm{V} 5$. We next performed an immunoprecipitation assay to isolate Beclin-1 protein and found that its ubiquitination levels significantly decrease in dependence of c-FLIP overexpression (Fig. 4C), leading us to conclude that $\mathrm{c}-\mathrm{FLIP}_{\mathrm{L}}$ is important to stabilize Beclin1 protein expression by reducing Beclin-1 proteasomal degradation.

Bioinformatics simulation and biochemical data correlate with a competition dynamic between C-FLIP $\mathrm{L}_{L}$ and NEDD4

As mentioned above, Beclin-1 is a pivotal member of the PI3K-III complex and its expression levels are tightly regulated via posttranslational modifications, which influence its activity on autophagy [37]. We thus used bioinformatic tools to simulate Beclin-1/c-FLIP interaction and found that the amino acids 349-352 of Beclin-1 are located at the interface region in the theoretical c-FLIP $/$ Beclin-1 complex, as shown in Fig. 5A. The 349-352 region constitutes Beclin1 PY motif (LPXY), which is a target of NEDD4 (neural precursor cellexpressed developmentally downregulated 4), a ubiquitin ligase that was shown to influence autophagy at multiple levels by interacting with several autophagic factors, including Vps34, p62 and Beclin-1 [48-51]. In particular, NEDD4 binds Beclin-1 via its PY motif, thus catalysing Beclin-1 polyubiquitination, an event that causes Beclin-1 degradation via the proteasome [52]. The position of the PY motif at the interface is also confirmed by the comparison of surface analysis on the monomeric structure and the complex (Fig. 5B). By studying, the solvent exposure of amino acids along the sequence revealed that the region 349-352 (and the following) in the complexed structure is different than in the monomeric structure. In more detail, this region results to be less accessible to the solvent in the complex than in the monomeric structure. This suggests that the predicted interaction of $\mathrm{c}-\mathrm{FLIP} \mathrm{P}_{\mathrm{L}}$ with Beclin-1 may mask the 349-352 segment, thus preventing Beclin-1 interaction with NEDD4. Moreover, on the 

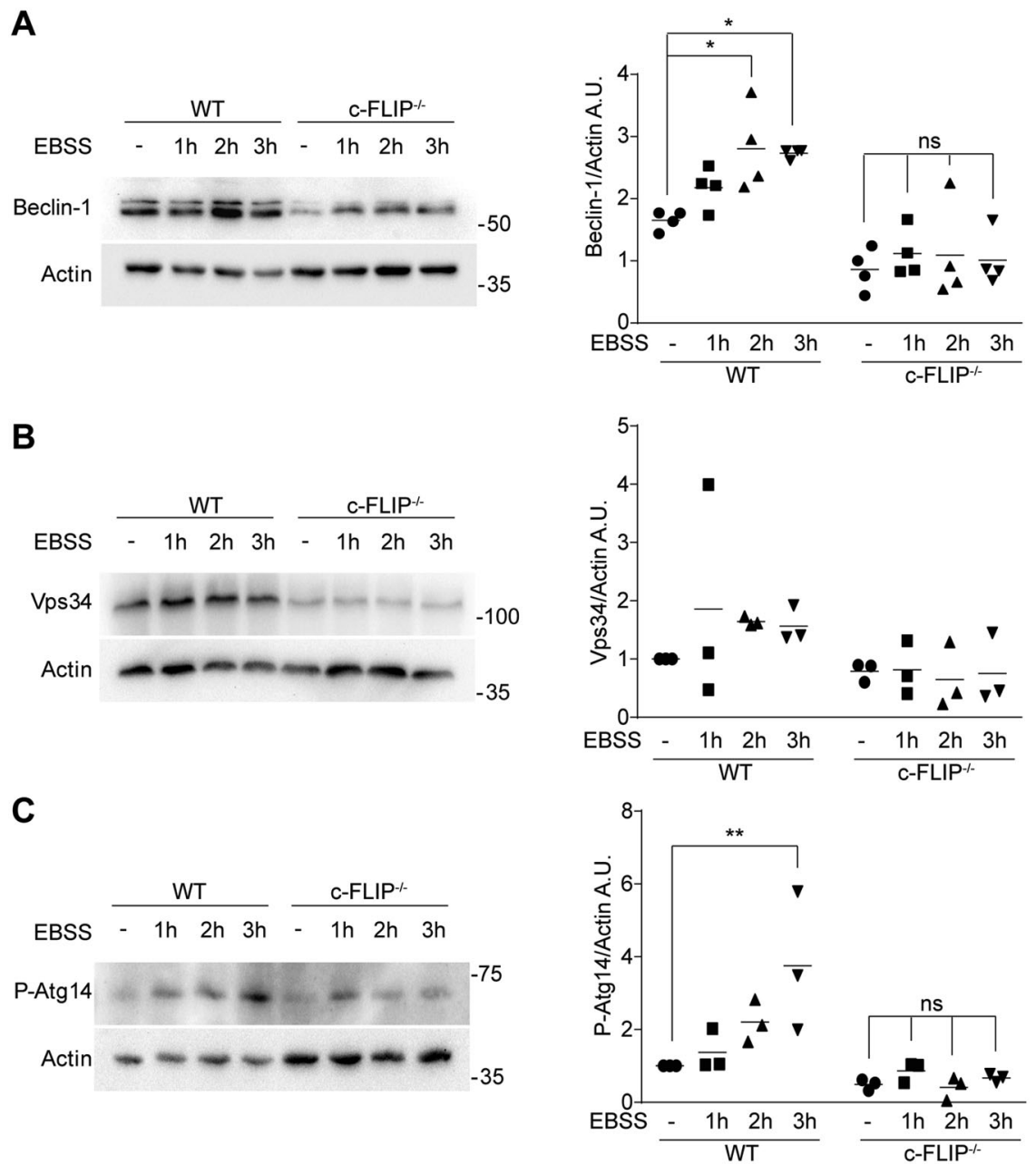

Fig. 3 WT and C-FLIP-/- MEFs were cultured in EBSS for increasing times (1, 2 and $3 \mathrm{~h})$ and the expression levels of Beclin-1, Vps34 and P-Atg14 were analyzed. A Beclin-1, B Vps34 and C P-Atg14 on Ser29 were studied by western blotting. $\beta$-Actin was used as a loading control. Data shown are representative of at least three individual experiments. ${ }^{*} p<0.05,{ }^{* *} p<0.01$ determined by two-way ANOVA.

other side of the complex, c-FLIP ${ }_{L}$ interacts with Beclin-1 via c-FLIP C-terminal portion, in particular via the segment formed by amino acids 463-479. To verify the hypothesis suggested by the bioinformatic analysis, we addressed c-FLIPL-NEDD4 competition dynamic by transiently transfecting HEK 293 cells with C-FLIP - V5and V5-NEDD4-expressing plasmids, and evaluating their effect on Beclin-1 protein stability. As shown in Fig. 5C, c-FLIP transfection enhances Beclin-1 protein levels as we previously showed. Combined c-FLIPL-NEDD4 transfection affects Beclin-1 levels, depending on the ratio of $c-F L I P_{L} / N E D D 4$ levels. As this ratio decreases, so does Beclin-1 expression level, therefore supporting the hypothesis that $\mathrm{C}-\mathrm{FLIP} \mathrm{P}_{\mathrm{L}}$ prevents NEDD4/Beclin-1 binding, thus reducing Beclin-1 proteasomal degradation. To further investigate this issue, we mutated Tyr352 of Beclin-1 into alanin (Y352A) according to previous evidence showing this amino acid substitution as capable to reduce Beclin-1 interaction with NEDD4 and consequently its ubiquitination [52]. Our experiments show that Beclin-1 Y352A mutant was, indeed, unaffected by C-FLIP $P_{L}$ transfection (Fig. 5D), indicating that Tyr352 is required for c-FLIP $\mathrm{L}^{-}$ dependent Beclin-1 stabilization and corroborating a competition dynamic between C-FLIP $\mathrm{L}$ and NEDD4.

\section{DISCUSSION}

In recent years, it became clear that the contribution of c-FLIP to cellular homoeostasis is complex, as c-FLIP controls different processes $[13,53,54]$. The involvement of c-FLIP in autophagy is a recent finding, which requires a deeper elucidation. In this work, we provide evidence for a direct influence of c-FLIP long isoform on autophagy.

Indeed, we here demonstrate that MEFs, which do not express c-FLIP, display a compromised autophagic flux in response to autophagy-inducing stimuli compared to WT cells (Fig. 1). Interestingly, when studying the autophagy induction stage, we found that both cell lines can trigger the process as mTOR kinase, the main endogenous inhibitor of autophagy, is inactivated upon starvation in both WT and c-FLIP-/- MEFs [43]. Analysis of the activation state of ULK1, a direct target of mTOR-mediated inhibitory phosphorylation, following a pro-autophagic stimulus confirmed that autophagy initiation happens regardless of c-FLIP protein presence (Fig. 2) [44].

Our group characterized C-FLIP as a modulator of ER morphology and ER-mitochondria crosstalk, showing that cFLIP localizes at ER and MAMs, and that c-FLIP-/- MEFs display an enlarged ER structure [12]. Remarkably, both ER and MAMs have been associated to omegasomes, the membranous structures from which autophagic vesicles originate [24]. Upon autophagy induction, the molecular machinery responsible for autophagosome nucleation migrates to omegasomes, where it mediates the production of Ptdlns $3 \mathrm{P}$ and induces membranes specialization into phagophores [26]. Thus, we investigated Beclin1 , Vps34 and P-Atg14 expression upon starvation in dependence 
A

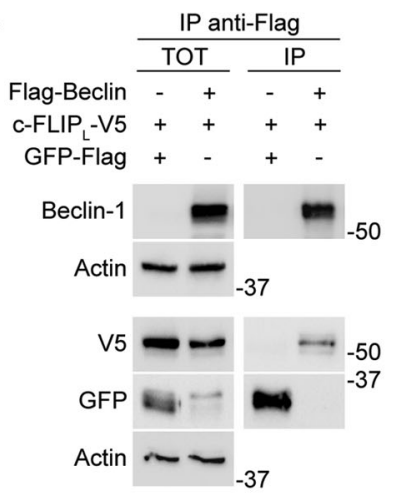



LIP
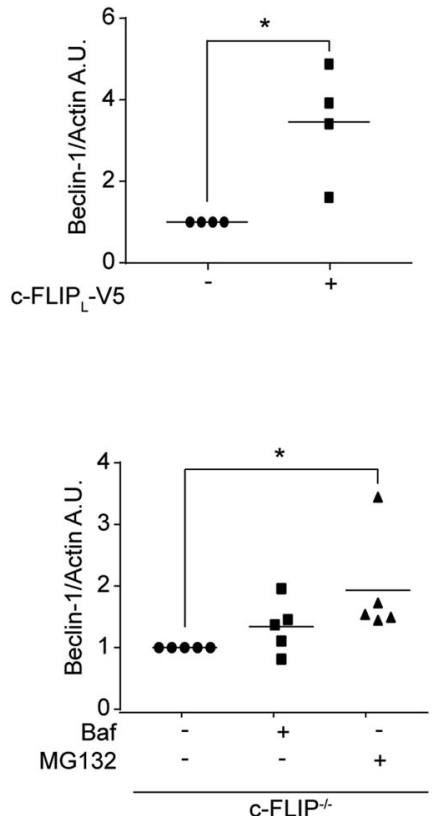

C



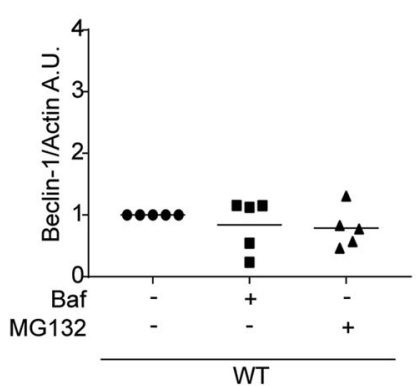


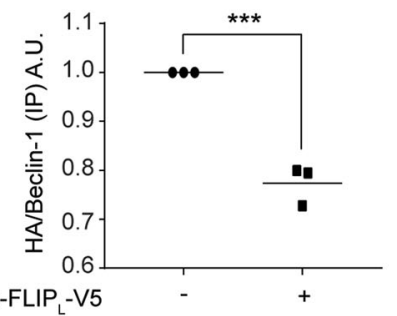

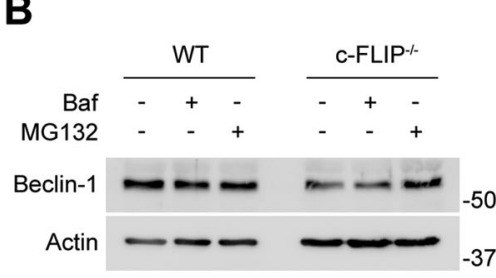

Fig. 4 c-FLIP interacts with Beclin-1 and affects Beclin-1 degradation. A Hek293 cells were transfected to overexpress c-FLIP (c-FLIP-V5) and Beclin-1 (Flag-Beclin). $\alpha$-Flag-beads were used to immunoprecipitate Beclin-1 and an $\alpha$-V5 antibody was used to immunoprecipitate c-FLIP. GFP-Flag was transfected to verify the specificity of the interaction. The result was detected by western blotting. $\beta$-Actin was used as a loading control. The graph quantifies Beclin-1 expression levels in correlation with c-FLIP overexpression. Data shown are representative of at least three individual experiments. ${ }^{*} p<0.05$ determined with two-tailed Student's $t$-test. B WT and $c$-FLIP-/- MEFs were treated with either bafilomycin (Baf) $(100 \mathrm{nM})$ or MG132 $(10 \mu \mathrm{M})$ for $4 \mathrm{~h}$ and Beclin-1 protein expression was then analysed by western blotting. Data shown are representative of at least three individual experiments. $\beta$-Actin was used as a loading control. ${ }^{*} p<0.05$ determined by one-way ANOVA. C Hek293 cells were transfected to overexpress c-FLIP (c-FLIPL-V5), Beclin-1 (Flag-Beclin) and ubiquitin (HA-Ubiquitin). $\alpha$-Flag-beads were used to immunoprecipitate Beclin-1. Protein signals were evaluated via western blotting and $\beta$-Actin was used as a loading control. Ubiquitination of Beclin-1 (HA/Beclin-1) was quantified in the graph. Data shown are representative of three individual experiments. ${ }^{* * *} p<0.001$ determined with two-tailed Student's $t$-test.

of c-FLIP. Selective binding of Atg14 to the core nucleation complex (Beclin-1-Vps34-Vps15) mediates its presence at the ER in the autophagosomes nucleation step [37]. We found that the levels of all the three markers increase after autophagy induction in WT MEFs, as expected, but not in c-FLIP-/- cells. We also found that all these proteins are downregulated in $c-F L I P-/-$ cells compared to WT in basal conditions as well (Fig. 3 and Supplementary Fig. S3).

c-FLIP is a scaffold protein devoid of any catalytic activity [10], which suggests that it may interact with the proteins involved in the autophagosome nucleation phase. All factors that participate to this step of the autophagic process are highly regulated and 
A

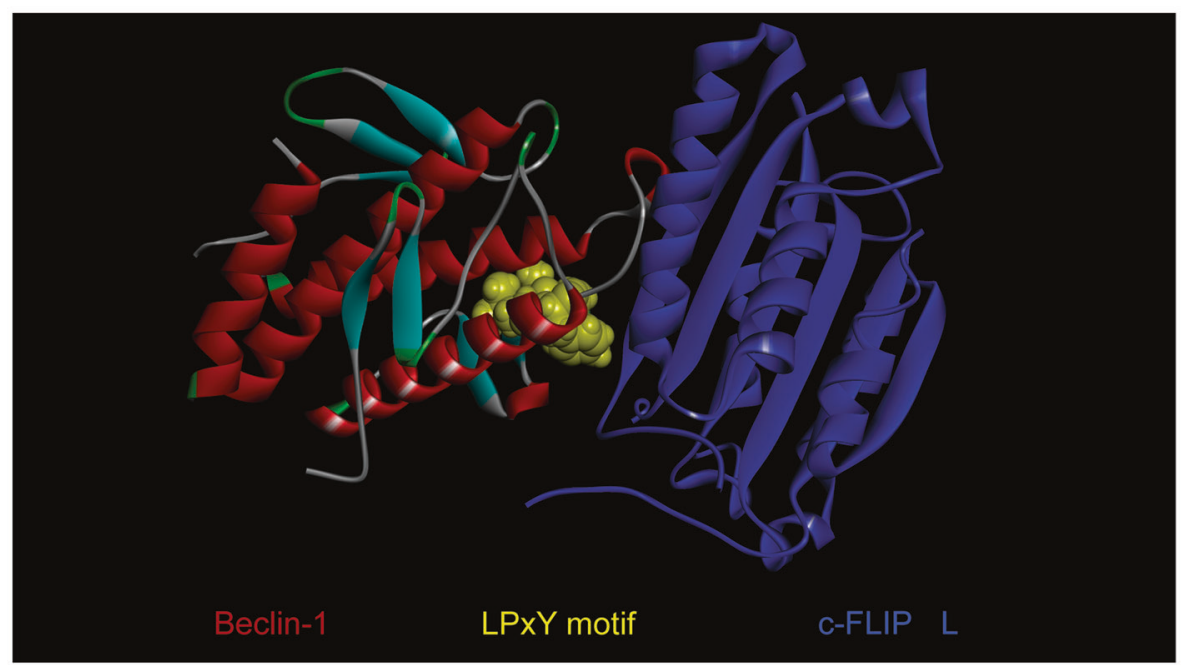

B
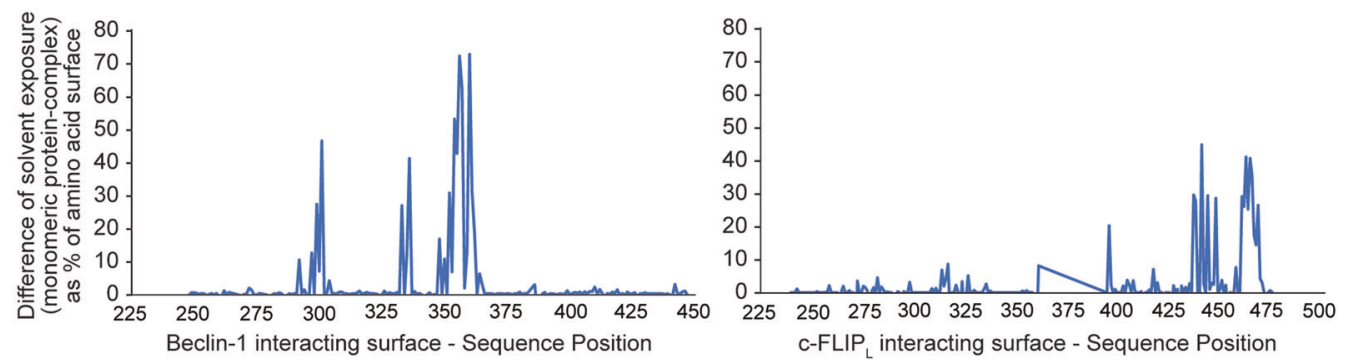

C

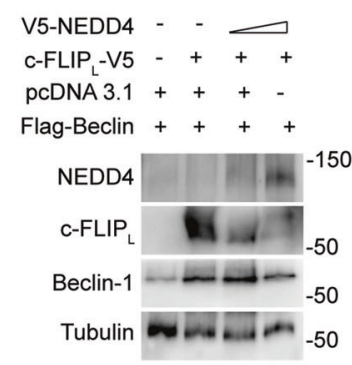

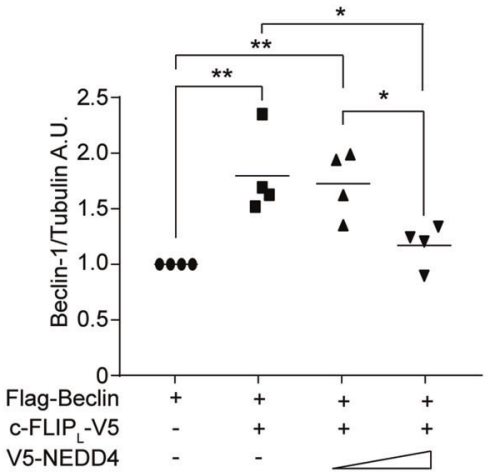

D

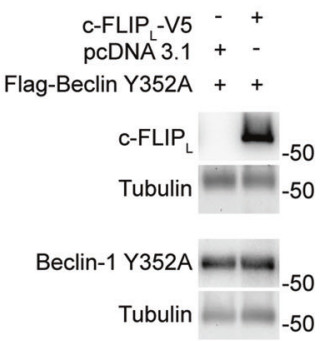

Fig. 5 Bioinformatics simulation and biochemical data correlate with a competition dynamic between c-FLIP $P_{L}$ and NEDD4. A The interaction between Beclin-1 and c-FLIP was simulated. Beclin-1 (on the left) is shown as a backbone ribbon with standard colours (red = helices, cyan $=\beta$-strand, green $=$ turn). The 349-352 region, which is reported in literature as being involved in the interaction with NEDD4, is highlighted in yellow. The region is near the surface of interaction with the c-FLIP molecule (on the right), shown with backbone ribbon in blue. B The graph evidences the sequence regions of Beclin-1 and of c-FLIP involved into the interaction between the two molecules as it reports the difference of solvent exposure of amino acids from the monomeric to the complex structure. Values $>0$ indicate amino acids that are solvent exposed in the monomeric structure and are not accessible, or less accessible, to the solvent in the complex, i.e., at the interface with the interacting molecule. C Hek293 cells were transfected to overexpress c-FLIP (c-FLIPL-V5), Beclin-1 (Flag-Beclin) and NEDD4 (V5NEDD4). Beclin-1 protein expression was then detected by western blotting. $\alpha$-Tubulin was used as a loading control. Data shown are representative of at least three individual experiments. ${ }^{*} p<0.05,{ }^{* *} p<0.01$ determined by one-way ANOVA. D Hek293 cells were transfected to overexpress c-FLIP (c-FLIPL-V5) and Beclin-1 mutant (Flag-Beclin Y352A). Beclin Y352A protein expression was studied by western blot analysis. $\alpha$-Tubulin was used as a loading control. Data shown are representative of three individual experiments.

strongly dependent on each other. In fact, the depletion of even only one of them compromises the entire complexes, as feedback mechanisms are called in action [26].

We here demonstrate that the absence of c-FLIP results in an alteration of the expression levels of some nucleation factors, i.e., Beclin-1 in particular (Fig. 3 and Supplementary Fig. S3). Therefore, we next explored a possible interaction between cFLIP $_{\mathrm{L}}$ and Beclin-1. The co-immunoprecipitation assays we performed shows that the two proteins interact in basal conditions. Furthermore, we noticed that Beclin-1 expression levels are affected by $C-F L I P_{L}$, both in endogenous and in overexpressing conditions in two different cellular models, suggesting that $\mathrm{C}-\mathrm{FLIP}_{\mathrm{L}}$ could influence Beclin-1 protein at a post-translational level. To investigate this issue, we treated WT and $c-F L I P-/-$ MEFs with MG132, a drug that blocks proteasome activity. In this way, we increased Beclin-1 levels in c-FLIP 
-/- cells, whereas proteasomal blockage did not affect the protein level in WT cells, suggesting that the lack of c-FLIP accelerates Beclin-1 turnover via proteasome. Furthermore, we show that $C-F L I P$ overexpression induces a reduction in Beclin-1 ubiquitination state, corroborating the theory of a highest degradation rate of Beclin-1 upon c-FLIP depletion and, therefore, that c-FLIP expression favours Beclin-1 protein stability (Fig. 4).

Finally, information obtained from our bioinformatic simulation suggested that the interaction between the C-terminal portion of C-FLIP $P_{L}$ and Beclin-1 involves Beclin-1 amino acids 349-352, which constitute the PY motif, recognized by the E3 ubiquitin ligase NEDD4 to label Beclin-1 for proteasomal degradation [52]. Interestingly, the C-terminal region of $\mathrm{C}-\mathrm{FLIP} \mathrm{P}_{\mathrm{L}}$ (amino acids from 463 to 479 ) is present only in the c-FLIP long isoform. These data well correlate with our biochemical analysis, which reveals a strong interaction between Beclin-1 and the long isoform of cFLIP, but not with the short one (Fig. 4 and Supplementary Fig. S4). Therefore, c-FLIP $\mathrm{L}_{\mathrm{L}}$ interaction with Beclin-1 may mask pivotal residues to the recognition of Beclin-1 by NEDD4 (Fig. 5A, B). Indeed, biochemical analyses revealed that NEDD4 overexpression counteracts C-FLIP $P_{L}$ effect on Beclin-1 stability, causing a decrease in Beclin-1 protein levels in a dose-dependent way (Fig. 5C). Moreover, Beclin-1 mutation of Tyr352 to alanine, which reportedly diminishes Beclin-1 ubiquitination and degradation

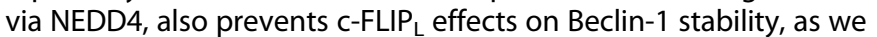
did not observe a difference of the Y352A mutant in dependence of C-FLIP $\mathrm{L}_{\mathrm{L}}$ overexpression (Fig. 5D). These results corroborated the concept of a competition dynamic between C-FLIP $\mathrm{L}_{\mathrm{L}}$ and NEDD4, which directly impacts Beclin-1 protein levels by regulating its turnover by the proteasomal pathway.

Our findings contribute to deepen the understanding of the complex crosstalk between autophagy and apoptosis. c-FLIP and Beclin-1 are key factors in the two pathways and they are both intriguing targets in anti-tumour therapies. As c-FLIP is traditionally considered as an anti-apoptotic protein, the goal is often to reduce its expression to induce apoptosis in cancer cells [53]. On the contrary, Beclin-1 is a haploinsufficient tumour suppressor, known to be downregulated in many cancer types [55]. As Beclin1 poor expression favours neoplastic transformations and tumour progression, the correlation we provide between these two proteins may pave the way to novel therapeutic paths. As it was widely demonstrated, balancing autophagy activation in cancer cells can influence their responses to therapies [56]. c-FLIP ${ }_{\mathrm{L}}$-Beclin1 -autophagy axis investigation may therefore open novel interesting scenarios to be exploited in cancer therapy.

\section{MATERIALS AND METHODS}

\section{Cell cultures}

WT and c-FLIP-/- MEFs were generous gifts of Tak W. Mak (Amgen Institute, Toronto, Canada). MEFs were cultured in Dulbecco's modified Eagle's medium (DMEM) (Sigma-Aldrich, Milano, Italy), enriched with 10\% fetal bovine serum $\left(\mathrm{Gibco}^{\mathrm{TM}}\right.$, Life Technologies Corporation, Grand Island, NY, USA), $2 \mathrm{mmol} / \mathrm{l}$ glutamine, $1 \mathrm{mmol} / \mathrm{l}$ sodium pyruvate and nonessential amino acids, in the presence of $100 \mathrm{U} / \mathrm{ml}$ penicillin and $100 \mu \mathrm{g} /$ $\mathrm{ml}$ streptomycin, all purchased from Sigma-Aldrich.

HEK 293 cells were cultured in DMEM enriched with 10\% fetal bovine serum in the presence of $100 \mathrm{U} / \mathrm{ml}$ penicillin and $100 \mu \mathrm{g} / \mathrm{ml}$ streptomycin.

Cells were maintained at $37{ }^{\circ} \mathrm{C}$ in a humidified $5 \% \mathrm{CO}_{2}$ atmosphere. All cell lines were tested for mycoplasma contamination prior to use.

\section{Cell treatments}

Cells were plated in $35 \mathrm{~mm}$ dishes for immunoblotting and immunofluorescence assays, and they were grown overnight. The day after plating, cells were treated for the indicated time. Torin 1 was purchased from Tocris Bioscience (Bristol, UK). Bafilomycin A1, tunicamycin, EBSS, 3-methyladenine and MG132 were purchased from Sigma-Aldrich.

\section{Cloning}

pcDNA3.1-Flag-BECN1.Y352A plasmid was generated using the Quick Change site-directed mutagenesis kit (Stratagene) on pcDNA3.1-FlagBECN1 plasmid using the following oligonucleotides: BECN1 Y352A_S, 5'TGCCGTTAGCCTGTTCTGGGGGGTTGCG-3'; BECN1 Y352A_AS, 5'-CCCAGAACAGGCTAACGGCAGCTCCTTAGATTTGTC-3'. Oligonucleotides were purchased by TAG Copenhagen (Denmark). Plasmid integrity was assessed by agarose gel electrophoresis and sequence confirmed by sequencing (Eurofin Genomics, Germany).

\section{Plasmids and transfection}

We used the following plasmids: Flag-Beclin, Flag-BeclinY352A, c-FLIPL-V5 (Thermo Fisher Scientific, Life Technologies Corporation), c-FLIP s-myc $^{-}$ (Thermo Fisher Scientific, Life Technologies Corporation), GFP-Flag, HAUbiquitin, V5-NEDD4 and pCDNA 3.1. V5-NEDD4 was a generous gift from Professor Daniela Rotin (University of Toronto, Canada). The day before transfection, cells were seeded to get the desired number of $80 \%$ confluence plates. Twenty-four hours later, cells were transfected using Lipofectamine2000 ${ }^{\circledR}$ (Invitrogen, San Giuliano Milanese, Italy) according to the manufacturer's guide.

\section{Cell lysis and protein quantification}

Cells were washed two times with pre-chilled phosphate-buffered saline (PBS) purchased from Sigma-Aldrich and were lysed.

In the case of MEFs, a commercial lysis Buffer 10x (Cell Signaling Technology, Danvers, MA, USA) complemented with $2 \%$ sodium dodecyl sulfate (SDS) and proteases' inhibitors (Sigma-Aldrich) was used. Cells were also sonicated (Branson Ultrasonic, Carouge, Switzerland) for $10 \mathrm{~s}$ at $50 \%$ amplitude.

In the case of HEK 293 cells, two different receipts were used as follows: (i) for the Beclin-1-c-FLIP co-immunoprecipitation: $50 \mathrm{mM}$ Tris-HCl pH 7.5, $150 \mathrm{mM} \mathrm{NaCl}, 1 \mathrm{mM}$ dithiothreitol, $0.5 \%$ Triton, $1 \mathrm{mM}$ EDTA pH 8, proteases and phosphatases inhibitors, all purchased from Sigma-Aldrich; (ii) for the ubiquitination and the competition assay: $50 \mathrm{mM}$ Tris- $\mathrm{HCl} \mathrm{pH} 7.5,1 \%$ Triton, $0.25 \%$ Na-Deoxycholate, $0.1 \%$ SDS, $150 \mathrm{mM} \mathrm{NaCl}, 1 \mathrm{mM}$ EDTA pH 8, $5 \mathrm{mM} \mathrm{MgCl}$, proteases and phosphatases inhibitors, all purchased from Sigma-Aldrich. Cells were then incubated for $30 \mathrm{~min}$ on ice.

Lysates from both cell lines were then centrifuged at $4{ }^{\circ} \mathrm{C}$ for $10 \mathrm{~min}$ at $13,000 \times g$ to remove cell debris.

Protein concentration was determined by micro BCA assay (Pierce, Rockford, IL, USA) and samples were boiled at $95^{\circ} \mathrm{C}$ for $10 \mathrm{~min}$ following Laemmli Buffer addition (0.04\% Bromophenol blue, 40\% Glycerol, $2 \%$ SDS, $20 \% \beta$-mercaptoethanol, $250 \mathrm{mM}$ Tris- $\mathrm{HCl} \mathrm{pH} .6 .8$, all purchased from Sigma-Aldrich.

\section{Co-immunoprecipitation}

Equal amounts of proteins $(300 \mu \mathrm{g})$ were used to perform the experiments. In the case of Flag-Beclin, proteins were incubated with pre-washed agarose beads conjugated with anti-Flag antibody (Sigma-Aldrich) for $1 \mathrm{~h}$ at $4{ }^{\circ} \mathrm{C}$ with rotation. In the case of C-FLIP $-\mathrm{V} 5$, proteins were incubated with $0.25 \mu \mathrm{g}$ of monoclonal anti-V5 antibody (Cell Signaling Technology) overnight at $4{ }^{\circ} \mathrm{C}$ with rotation. The next day, pre-washed magnetic beads (Dynabeads ${ }^{\circledast}$ Protein G, Novex ${ }^{\circledast}$, Life Technologies Corporation) were added to the mix and incubated for $1 \mathrm{~h}$ at $4{ }^{\circ} \mathrm{C}$ with rotation. In the case of c-FLIP, proteins were incubated with $0.25 \mu \mathrm{g}$ of monoclonal anti-FLIP antibody (Cell Signaling Technology) overnight at $4{ }^{\circ} \mathrm{C}$ with rotation. The next day, pre-washed agarose beads (GE Healthcare, Chicago, IL, USA) were added to the mix and incubated for $1 \mathrm{~h}$ at $4{ }^{\circ} \mathrm{C}$ with rotation.

Samples were washed four times by centrifugation $(5 \mathrm{~min}, 2500 \times \mathrm{g})$ with washing buffer (lysis buffer receipt without protease and phosphatase inhibitors) to collect the beads. The proteins bound to the beads were eluted in $26 \mu$ l of $2 \times$ Laemmli Buffer and boiled.

\section{Ubiquitination assay}

Equal amounts of proteins $(300 \mu \mathrm{g})$ were used to perform the experiments.

Proteins were incubated with pre-washed agarose beads conjugated with anti-Flag antibody for $1 \mathrm{~h}$ at $4{ }^{\circ} \mathrm{C}$ with rotation. After the lysis and prior incubation with anti-Flag antibody, $1 \%$ SDS was added to the lysates, which were incubated at $90^{\circ} \mathrm{C}$ for $5 \mathrm{~min}$ to dissociate protein-protein interactions as previously described [57]. Immunoprecipitation buffer was added to the lysates to tenfold dilute the samples. The immunoprecipitation was then carried on as described above. 


\section{Immunoblotting}

Proteins were separated by SDS-polyacrylamide gel electrophoresis and transferred on either polyvinylidene fluoride membranes or nitrocellulose membranes (Amersham Bioscience, Piscataway, NJ, USA). Membranes were probed using the following antibodies: anti-p62 (Abcam, Cambridge, UK); anti-Atg5, anti-P-Atg14 (Ser29), anti-Beclin-1, anti-FLIP, anti-LC3, antiNEDD4, anti-mTOR, anti-P-mTOR (Ser2448), anti-ULK1, anti-P-ULK1 (Ser757), anti-V5, anti-Vps34 (Cell Signaling Technology); anti-BECN1, anti-GFP, anti-HA (Santa Cruz Biotechnology, Heidelberg, Germany); and anti-B-Actin, anti-Tubulin (Sigma-Aldrich). Secondary antibodies were horseradish peroxidase-conjugated anti-mouse or anti-rabbit (Bio-rad, Hercules, CA, USA). Membranes were washed with Tris-buffered saline (Medicago, Danmarks-Berga, Uppsala, Sweden) with $0.1 \%$ Tween-20 (Sigma-Aldrich) and developed through the chemiluminescence system (Amersham Bioscience) on the ChemiDock image analyser (Bio-Rad), which was also used for densitometric quantifications.

\section{Immunofluorescence}

Cells were washed in PBS and fixed with 3.7\% paraformaldehyde (Electron Microscopy Sciences, Industry Road Hatfield, PA, USA), followed by permeabilization with $0.4 \%$ Triton X-100 (Sigma-Aldrich) in PBS for $5 \mathrm{~min}$. The samples were then blocked with $5 \%$ goat serum (Sigma-Aldrich) plus $0.3 \%$ Triton X-100 for $1.5 \mathrm{~h}$ and then incubated overnight with the primary antibody rabbit anti-LC3 (Cell Signaling Technology) according to the supplier's indications. Following three washes of $5 \mathrm{~min}$ each with PBS, secondary goat anti-rabbit IgG-FITC antibody (Sigma-Aldrich) was added to the samples for $1 \mathrm{~h}$. TO-PRO-3 lodide (642/661) (Thermo Fisher Scientific, Life Technologies Corporation) was used to stain the nuclei according to the manufacturer's instructions. As a control for immunofluorescence, the primary antibody was omitted and no fluorescence was detected under these conditions.

Pictures were acquired using a Leica TCS SP2 confocal microscope with a $\times 40$ oil-immersion objective. Images were assembled in panels and puncta were counted using Fiji software (ImageJ Development Team, Universal Imaging Corporation, West Chester, PA, USA).

\section{Bioinformatics}

The molecular interaction of Beclin-1 and $\mathrm{C}-\mathrm{FLIP} \mathrm{P}_{\mathrm{L}}$ was simulated by using the Schrödinger's BioLuminate software (https://www.schrodinger.com/ products/bioluminate). Standard procedure described in the manual for protein-protein docking was applied. The molecular model used for Beclin-1 is the crystal structure of Beclin-1 evolutionary conserved domain, PDB structure 4DDP [58]. The molecular model used for c-FLIP is the crystal structure deposited in PDB as $3 \mathrm{H} 13$ [59]. Image of the simulated complex between Beclin-1 and c-FLIP was generated with the Discovery Studio software [60] on the monomeric proteins and on the complex.

\section{Statistical analysis}

All statistical analysis was performed using Prism software (GraphPad Software, San Diego, CA, USA). Values are expressed as mean, with individual experiments data points plotting. The statistical significance was determined by performing two-tailed Student's $t$-test, one-way or two-way analysis of variance, depending on the number of variables involved in each experiment; a value of $P \leq 0.05$ was considered statistically significant.

\section{REFERENCES}

1. Thome M, Schneider $P$, Hofmann K, Fickenscher $H$, Meinl E, Neipel F, et al. Viral FLICE-inhibitory proteins (FLIPs) prevent apoptosis induced by death receptors. Nature 1997;386:517-21.

2. Irmler $M$, Thome $M$, Hahne $M$, Schneider $P$, Hofmann $K$, Steiner V, et al. Inhibition of death receptor signals by cellular FLIP. Nature 1997;388:190-5.

3. Kataoka T, Budd RC, Holler N, Thome M, Martinon F, Irmler M, et al. The caspase-8 inhibitor FLIP promotes activation of NF-kappaB and Erk signaling pathways. Curr Biol. 2000;10:640-8.

4. Yeh WC, Itie A, Elia AJ, Ng M, Shu HB, Wakeham A, et al. Requirement for Casper (c-FLIP) in regulation of death receptor-induced apoptosis and embryonic development. Immunity 2000;12:633-42.

5. Giampietri C, Petrungaro S, Musumeci M, Coluccia P, Antonangeli F, De Cesaris $P$, et al. c-Flip overexpression reduces cardiac hypertrophy in response to pressure overload. J Hypertension. 2008;26:1008-16.
6. Giampietri C, Petrungaro S, Coluccia P, Antonangeli F, Giannakakis K, Faraggiana $\mathrm{T}$, et al. c-Flip overexpression affects satellite cell proliferation and promotes skeletal muscle aging. Cell Death Dis. 2010;1:e38.

7. Lens SM, Kataoka T, Fortner KA, Tinel A, Ferrero I, MacDonald RH, et al. The caspase 8 inhibitor C-FLIP $(\mathrm{L})$ modulates T-cell receptor-induced proliferation but not activation-induced cell death of lymphocytes. Mol Cell Biol. 2002;22:5419-33.

8. Schattenberg JM, Zimmermann T, Worns M, Sprinzl MF, Kreft A, Kohl T, et al. Ablation of c-FLIP in hepatocytes enhances death-receptor mediated apoptosis and toxic liver injury in vivo. J Hepatol. 2011;55:1272-80.

9. Ueffing N, Keil E, Freund C, Kuhne R, Schulze-Osthoff K, Schmitz I. Mutational analyses of c-FLIPR, the only murine short FLIP isoform, reveal requirements for DISC recruitment. Cell Death Differ. 2008;15:773-82.

10. Ozturk S, Schleich K, Lavrik IN. Cellular FLICE-like inhibitory proteins (c-FLIPs): finetuners of life and death decisions. Exp Cell Res. 2012;318:1324-31.

11. Zhang J, Chen Y, Huang Q, Cheng W, Kang Y, Shu L, et al. Nuclear localization of C-FLIP-L and its regulation of AP-1 activity. Int J Biochem Cell Biol. 2009;41:1678-84.

12. Marini ES, Giampietri C, Petrungaro S, Conti S, Filippini A, Scorrano L, et al. The endogenous caspase-8 inhibitor c-FLIPL regulates ER morphology and crosstalk with mitochondria. Cell Death Differ. 2015:22:1131-43.

13. Fricker N, Beaudouin J, Richter P, Eils R, Krammer PH, Lavrik IN. Model-based dissection of CD95 signaling dynamics reveals both a pro- and antiapoptotic role of c-FLIPL. J Cell Biol. 2010;190:377-89.

14. Smyth P, Sessler T, Scott CJ, Longley DB. FLIP(L): the pseudo-caspase. FEBS J. 2020;287:4246-60.

15. Mizushima N. A brief history of autophagy from cell biology to physiology and disease. Nat Cell Biol. 2018;20:521-7.

16. Tomaipitinca L, Mandatori S, Mancinelli R, Giulitti F, Petrungaro S, Moresi V. et al. The role of autophagy in liver epithelial cells and its impact on systemic homeostasis. Nutrients. 2019;11:827.

17. Saha S, Panigrahi DP, Patil S, Bhutia SK. Autophagy in health and disease: a comprehensive review. Biomed Pharmacother. 2018;104:485-95.

18. Aburto MR, Hurle JM, Varela-Nieto I, Magarinos M. Autophagy during vertebrate development. Cells 2012;1:428-48.

19. Scrivo A, Bourdenx M, Pampliega O, Cuervo AM. Selective autophagy as a potential therapeutic target for neurodegenerative disorders. Lancet Neurol. 2018;17:802-15.

20. Marinkovic M, Sprung M, Buljubasic M, Novak I. Autophagy modulation in cancer: current knowledge on action and therapy. Oxid Med Cell Longev. 2018;2018:8023821.

21. Galluzzi L, Vitale I, Aaronson SA, Abrams JM, Adam D, Agostinis P, et al. Molecular mechanisms of cell death: recommendations of the Nomenclature Committee on Cell Death 2018. Cell Death Differ. 2018;25:486-541.

22. D'Arcangelo D, Giampietri C, Muscio M, Scatozza F, Facchiano F, Facchiano A WIPI1, BAG1, and PEX3 autophagy-related genes are relevant melanoma markers. Oxid Med Cell Longev. 2018;2018:1471682.

23. Giampietri C, Petrungaro S, Cordella M, Tabolacci C, Tomaipitinca L, Facchiano A. et al. Lipid storage and autophagy in melanoma cancer cells. Int J Mol Sci. 2017;18:1271.

24. Carlsson SR, Simonsen A. Membrane dynamics in autophagosome biogenesis. J Cell Sci. 2015;128:193-205.

25. Paquette $\mathrm{M}$, El-Houjeiri L, Pause A. mTOR pathways in cancer and autophagy. Cancers 2018;10:1.

26. Mercer TJ, Gubas A, Tooze SA. A molecular perspective of mammalian autophagosome biogenesis. J Biol Chem. 2018;293:5386-95.

27. Lee YK, Lee JA. Role of the mammalian ATG8/LC3 family in autophagy: differential and compensatory roles in the spatiotemporal regulation of autophagy. BMB Rep. 2016;49:424-30.

28. Nakamura S, Yoshimori T. New insights into autophagosome-lysosome fusion. J Cell Sci. 2017;130:1209-16.

29. Lee JS, Li Q, Lee JY, Lee SH, Jeong JH, Lee HR, et al. FLIP-mediated autophagy regulation in cell death control. Nat Cell Biol. 2009;11:1355-62.

30. Bell BD, Leverrier S, Weist BM, Newton RH, Arechiga AF, Luhrs KA, et al. FADD and caspase-8 control the outcome of autophagic signaling in proliferating $T$ cells. Proc Natl Acad Sci USA. 2008;105:16677-82.

31. Park MA, Zhang G, Norris J, Hylemon PB, Fisher PB, Grant $S$, et al. Regulation of autophagy by ceramide-CD95-PERK signaling. Autophagy 2008;4:929-31.

32. Conti S, Petrungaro S, Marini ES, Masciarelli S, Tomaipitinca L, Filippini A, et al. A novel role of c-FLIP protein in regulation of ER stress response. Cell Signal. 2016;28:1262-9.

33. Mizushima N. Autophagy: process and function. Genes Dev. 2007;21:2861-73.

34. Itakura E, Kishi C, Inoue K, Mizushima N. Beclin 1 forms two distinct phosphatidylinositol 3-kinase complexes with mammalian Atg14 and UVRAG. Mol Biol Cell. 2008;19:5360-72. 
35. Fimia GM, Stoykova A, Romagnoli A, Giunta L, Di Bartolomeo S, Nardacci R, et al. Ambra1 regulates autophagy and development of the nervous system. Nature 2007;447:1121-5.

36. Matsunaga $K$, Saitoh $T$, Tabata $K$, Omori H, Satoh T, Kurotori N, et al. Two Beclin 1binding proteins, Atg14L and Rubicon, reciprocally regulate autophagy at different stages. Nat Cell Biol. 2009;11:385-96.

37. Boutouja F, Brinkmeier R, Mastalski T, El Magraoui F, Platta HW. Regulation of the tumor-suppressor BECLIN 1 by distinct ubiquitination cascades. Int J Mol Sci. 2017;18:2541.

38. Sun $H$, Yu J, Wen Z, Wang M, Chen W. Decreased expression of Beclin-1 in patients with hepatocellular carcinoma. J BUON. 2019;24:634-41.

39. Wang $\Pi$, Cao $\mathrm{QH}$, Chen MY, Xia Q, Fan XJ, Ma XK, et al. Beclin 1 deficiency correlated with lymph node metastasis, predicts a distinct outcome in intrahepatic and extrahepatic cholangiocarcinoma. PLoS ONE. 2013;8:e80317.

40. Bhardwaj M, Leli NM, Koumenis C, Amaravadi RK. Regulation of autophagy by canonical and non-canonical ER stress responses. Semin Cancer Biol. 2020;66:116-28.

41. Klionsky DJ, Abdel-Aziz AK, Abdelfatah S, Abdellatif M, Abdoli A, Abel S, et al. Guidelines for the use and interpretation of assays for monitoring autophagy (4th edition)(1). Autophagy 2021;17:1-382.

42. Romanov J, Walczak M, Ibiricu I, Schuchner S, Ogris E, Kraft C, et al. Mechanism and functions of membrane binding by the Atg5-Atg12/Atg16 complex during autophagosome formation. EMBO J. 2012;31:4304-17.

43. Holz MK, Blenis J. Identification of S6 kinase 1 as a novel mammalian target of rapamycin (mTOR)-phosphorylating kinase. J Biol Chem. 2005;280:26089-93.

44. Kim J, Kundu M, Viollet B, Guan KL. AMPK and mTOR regulate autophagy through direct phosphorylation of Ulk1. Nat Cell Biol. 2011;13:132-41.

45. Park JM, Jung CH, Seo M, Otto NM, Grunwald D, Kim KH, et al. The ULK1 complex mediates MTORC1 signaling to the autophagy initiation machinery via binding and phosphorylating ATG14. Autophagy 2016;12:547-64.

46. Wirawan E, Vande Walle L, Kersse K, Cornelis S, Claerhout S, Vanoverberghe I, et al. Caspase-mediated cleavage of Beclin-1 inactivates Beclin-1-induced autophagy and enhances apoptosis by promoting the release of proapoptotic factors from mitochondria. Cell Death Dis. 2010;1:e18.

47. Lee DH, Goldberg AL. Proteasome inhibitors: valuable new tools for cell biologists. Trends Cell Biol. 1998;8:397-403.

48. Xie W, Jin S, Wu Y, Xian H, Tian S, Liu DA, et al. Auto-ubiquitination of NEDD4-1 Recruits USP13 to facilitate autophagy through deubiquitinating VPS34. Cell Rep. 2020;30:2807-19.e4.

49. Lin Q, Dai Q, Meng H, Sun A, Wei J, Peng K, et al. The HECT E3 ubiquitin ligase NEDD4 interacts with and ubiquitylates SQSTM1 for inclusion body autophagy. J Cell Sci. 2017;130:3839-50.

50. Huang X, Chen J, Cao W, Yang L, Chen Q, He J, et al. The many substrates and functions of NEDD4-1. Cell Death Dis. 2019;10:904.

51. Pei G, Buijze H, Liu H, Moura-Alves P, Goosmann C, Brinkmann V, et al. The E3 ubiquitin ligase NEDD4 enhances killing of membrane-perturbing intracellular bacteria by promoting autophagy. Autophagy 2017;13:2041-55.

52. Platta HW, Abrahamsen $\mathrm{H}$, Thoresen SB, Stenmark H. Nedd4-dependent lysine11-linked polyubiquitination of the tumour suppressor Beclin 1. Biochem. J. 2012;441:399-406.

53. Humphreys L, Espona-Fiedler M, Longley DB. FLIP as a therapeutic target in cancer. FEBS J. 2018;285:4104-23.

54. Giampietri C, Petrungaro S, Klinger FG, Coluccia P, Paone A, Vivarelli E, et al. c-Flip expression and function in fetal mouse gonocytes. FASEB J. 2006;20:124-6.

55. Yue Z, Jin S, Yang C, Levine AJ, Heintz N. Beclin 1, an autophagy gene essential for early embryonic development, is a haploinsufficient tumor suppressor. Proc Natl Acad Sci USA. 2003;100:15077-82.

56. Galluzzi L, Pietrocola F, Bravo-San Pedro JM, Amaravadi RK, Baehrecke EH, Cecconi $\mathrm{F}$, et al. Autophagy in malignant transformation and cancer progression. EMBO J. 2015;34:856-80.

57. Nazio F, Strappazzon F, Antonioli M, Bielli P, Cianfanelli V, Bordi M, et al. mTOR inhibits autophagy by controlling ULK1 ubiquitylation, self-association and function through AMBRA1 and TRAF6. Nat Cell Biol. 2013;15:406-16.

58. Huang W, Choi W, Hu W, Mi N, Guo Q, Ma M, et al. Crystal structure and biochemical analyses reveal Beclin 1 as a novel membrane binding protein. Cell Res. 2012;22:473-89.

59. Yu JW, Jeffrey PD, Shi Y. Mechanism of procaspase-8 activation by c-FLIPL. Proc Natl Acad Sci USA. 2009;106:8169-74.
60. Hubbard SJ, Campbell SF, Thornton JM. Molecular recognition. Conformational analysis of limited proteolytic sites and serine proteinase protein inhibitors. J Mol Biol. 1991;220:507-30.

\section{ACKNOWLEDGEMENTS}

We thank Tak W. Mak for providing WT and C-FLIP-/- MEFs, and Daniela Rotin for providing the V5-NEDD4 plasmid. We also thank Federica Barbagallo, Giovanni Bernardini, Valentina Cianfanelli, Antonio Facchiano, Francesca Nazio, Simona Polo and Elena Vicini for their helpful suggestions. Furthermore, we thank Stefania De Grossi for her technical support at the confocal microscope.

\section{AUTHOR CONTRIBUTIONS}

L.T. conceived, planned and carried out the experiments, and wrote the manuscript. S.P. conceived and carried out the experiments. P.D. and S.R. performed cloning experiments and provided critical feedback. A.F. and A.D. planned and carried out the bioinformatics simulations. F.G. contributed to sample preparation. E.G., A.F. and E.Z. helped supervise the project. F.C. contributed to the design and planning of the research. C.G. planned, supervised the work and contributed to the final version. All authors discussed and agreed upon the final manuscript.

\section{FUNDING STATEMENT}

This research was funded by Ricerca Scientifica Sapienza grants, by Cooperation with developing countries Sapienza 2017 and Fondo di Finanziamento delle Attività Base della Ricerca grants.

\section{ETHICS STATEMENT}

Ethics approval was not required for this work.

\section{COMPETING INTERESTS}

The authors declare no competing interests.

\section{ADDITIONAL INFORMATION}

Supplementary information The online version contains supplementary material available at https://doi.org/10.1038/s41419-021-03957-5.

Correspondence and requests for materials should be addressed to A.F. or C.G.

Reprints and permission information is available at http://www.nature.com/ reprints

Publisher's note Springer Nature remains neutral with regard to jurisdictional claims in published maps and institutional affiliations.

Open Access This article is licensed under a Creative Commons Attribution 4.0 International License, which permits use, sharing, adaptation, distribution and reproduction in any medium or format, as long as you give appropriate credit to the original author(s) and the source, provide a link to the Creative Commons license, and indicate if changes were made. The images or other third party material in this article are included in the article's Creative Commons license, unless indicated otherwise in a credit line to the material. If material is not included in the article's Creative Commons license and your intended use is not permitted by statutory regulation or exceeds the permitted use, you will need to obtain permission directly from the copyright holder. To view a copy of this license, visit http://creativecommons. org/licenses/by/4.0/.

(c) The Author(s) 2021 